\title{
Microwave Properties of Ferromagnetic Nanowires and Applications to Tunable Devices
}

\author{
J. Spiegel ${ }^{\mathrm{a}}$ and I. Huynen ${ }^{\mathrm{b}}$ \\ Université Catholique de Louvain, Microwave Laboratory, Place du Levant 3, \\ B-1348 Louvain-la-neuve, Belgium \\ ajudith.spiegel@uclouvain.be, ${ }^{\mathrm{b}}$ isabelle.huynen@uclouvain.be
}

\begin{abstract}
Keywords: Ferromagnetic nanowires, RF characterization, magnetodielectric effect.
Abstract. Microwave devices as circulators or tunable filters demand nowadays small size and broad bandwidth. Ferromagnetic nanowired membranes are ideal candidates for this purpose. This paper focuses on the dielectric properties of such substrates, as influenced by the ferromagnetic nature of nanowires and their filling factor. Two particular cases are considered: a membrane filled up to its top with nanowires, forming a one-layer substrate, and a membrane filled up to a certain percentage of its height with nanowires, forming a two-layer substrate. The models proposed in this paper for each case take the inductive and gyromagnetic effects in the wires into account. They predict for the one-layer case a magnetodielectric behavior which is tunable by applying an external magnetic field. The effect is no longer visible for the two-layer topology corresponding to microwave circuit applications.
\end{abstract}

\section{Introduction}

Ferromagnetic nanowired substrates (Fig. 1a) offer, due to their excellent material properties, the ability to build non-reciprocal or tunable planar devices for very high frequencies, as nowadays requested for wireless communication and automotive systems. The advantages of such materials compared to classical ferrites are a higher operation frequency, a higher saturation magnetization and a higher resonant frequency. In addition their properties are tunable by applying an external magnetic field [1]. The nanowires as ferromagnetic inclusions influence the permittivity and permeability of the composite substrate. For the development of nanowired devices, the dielectric and magnetic properties of the substrate have to be known very exactly.

The focus of this paper lies on the dielectric properties of nanowired substrates as influenced by their magnetic and gyrotropic properties. We developed a theoretical permittivity model, which takes the internal and external inductances of the wires as well as geometrical and material parameters of the artificial substrate into account. The influence of the porosity, the height and diameter of the nanowires has been studied for two particular substrates: a two-layer substrate formed by a membrane filled up to a certain percentage of its height with nanowires, and a one-layer substrate completely filled with nanowires. Under specific conditions, the one-layer substrate shows a tunable magnetodielectric behavior in the microwave range.

\section{Topology of ferromagnetic nanowired substrate}

The so-called "nanowired substrate" of Fig. 1a consists of a porous insulating polycarbonate or alumina membrane filled with ferromagnetic nanowires by electrodeposition. The cylindrical pores of diameter $\mathrm{D}$ are randomely distributed and electromagnetic waves can propagate untroubled, when the diameter of the nanowires is smaller than skindepth [2].

As already mentioned, we studied two topologies, one is a two-layer substrate, where the membrane is filled up to a certain percentage of its height with nanowires, so that there is one sublayer containing nanowires and the other contains no nanowires, air is in the pores. The one-layer substrate is based on a membrane filled completely with nanowires. The two-layer substrate corresponds to the actual topology of planar microwave devices. For them, an insulating top layer is 
necessary to avoid short-circuits/contacts between the top of the nanowires and the top microstrip conductor. This topology is more application-oriented and easy to characterize experimentally by transmission measurements of RF signals guided in the substrate by the microstrip line [2]. The onelayer approach can be validated by cavity perturbation measurements avoiding short-circuits since no planar conductor is needed.

Our permittivity model previously developed from a microstrip approach [2] is extended here to take the magnetodielectric effect into account. Fig. 1b shows the equivalent circuit of the substrate, based on capacitances per unit length for a microstrip line including nanowires in the supporting substrate. It includes the internal magnetic properties of the wires to control the effective permittivity. These properties can be deduced from our permeability model proposed in [3]. From this equivalent circuit, we will derive later in this paper the permittivity model for the one- and twolayer cases.

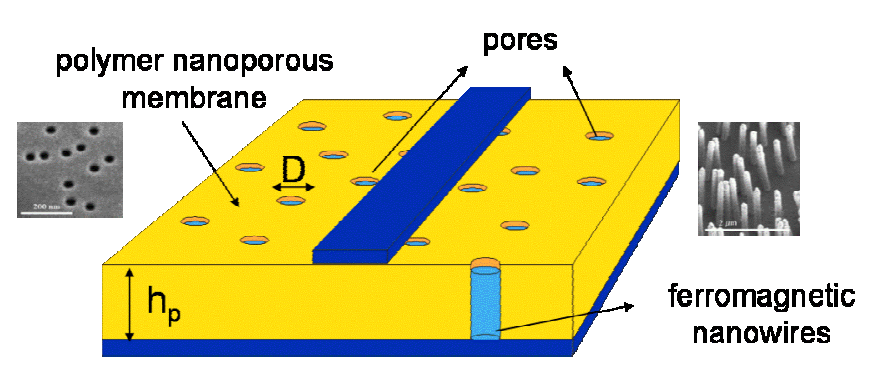

(a)

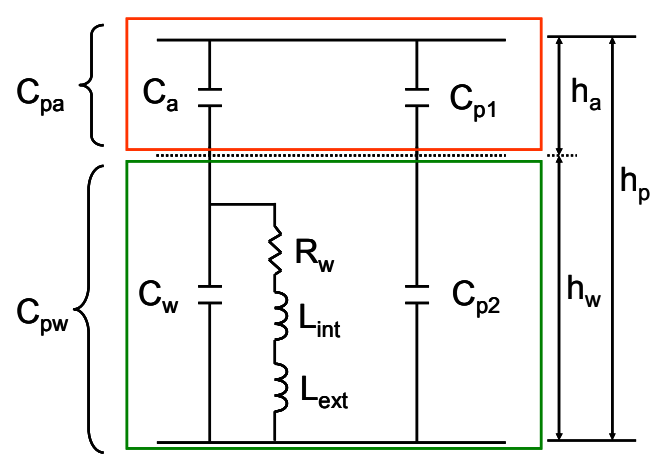

(b)

Fig. 1: (a) Ferromagnetic nanowired substrate, (b) equivalent circuit of capacitance per unit length for a microstrip line including nanowires in the supporting substrate.

\section{Permeability Model}

Our permeability model is deduced from the calculation of the effective propagation constant $\gamma_{\mathrm{eff}}$ of the two layer substrate by a variational model [4]. The effective permeability results from

$$
\mu_{\text {eff }}=\left(\frac{\gamma_{\text {eff }}}{j \omega}\right)^{2} \cdot c^{2},
$$

where $\mathrm{c}$ is the speed of light in the dielectric substrate, i. e. a substrate where only the conductive nature of the metallic wire is taken into account. Details of the models are given in [3-4].

This effective permeability model has been validated by measurements and is shown in Fig. 2. Fig. $2 \mathrm{a}$ shows the real part and Fig. $2 \mathrm{~b}$ the imaginary part of $\mu_{\text {eff. }}$ The membrane has a low porosity of $3 \%$ and the nanowire diameter is $50 \mathrm{~nm}$. Both curves show the typical behavior of ferromagnetic nanowires in a low porosity membrane. The magnetic response is weak, but visible in the range of the ferromagnetic resonance (FMR).

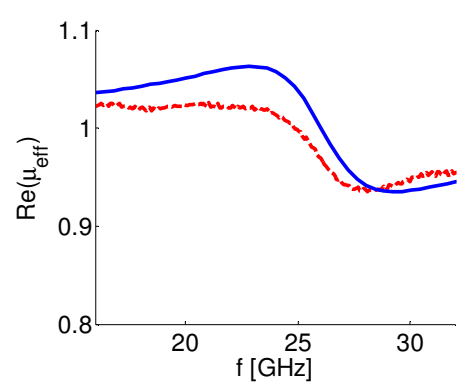

(a)

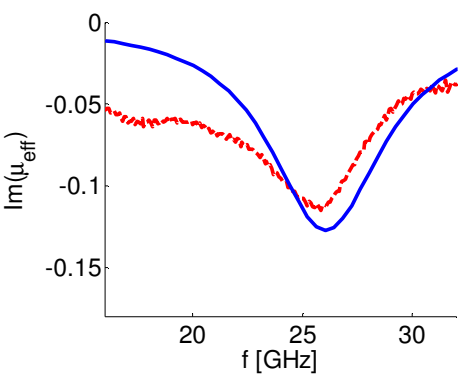

(b)

Fig. 2: Simulation (solid) and measurement (dashed) of effective permeability of two-layer substrate based on microstrip approach with porosity $\mathrm{p}=3 \%$ and wire diameter of $50 \mathrm{~nm}$ : (a) real part and (b) imaginary part. 


\section{Extended Permittivity Model}

The two-layer substrate, shown in Fig. 1b, is based on a microstrip line capacitance approach. The upper layer capacitance, designated $\mathrm{C}_{\mathrm{pa}}$, contains the parallel connection of $\mathrm{C}_{\mathrm{a}}$, the air capacitance and $\mathrm{C}_{\mathrm{p} 1}$, the polymer capacitance between the top of the ferromagnetic nanowire and the bottom of the microstrip line. The lower layer capacitance, designated $\mathrm{C}_{\mathrm{pw}}$, contains the parallel connection of $\mathrm{C}_{\mathrm{w}}$, the wire capacitance, $\mathrm{C}_{\mathrm{p} 2}$, the polymer capacitance, and $\mathrm{R}_{\mathrm{w}}$, wire resistance with $\mathrm{L}_{\mathrm{int}}$, internal and $\mathrm{L}_{\text {ext }}$ external inductance of the wire. From the series connection of these two layers capacitances, the effective permittivity results in:

$$
\varepsilon_{e f f}=\frac{C_{t o t} \cdot h_{p}}{\varepsilon_{0} W_{s}}=\frac{h_{p}}{\frac{h_{w}}{\varepsilon_{w}}+\frac{h_{a}}{\varepsilon_{p}}},
$$

where $h_{p}$ is the height of the membrane, $h_{w}$ the height of the nanowires and $h_{a}$ the height between the top of the nanowire and the bottom of the microstrip line, see Fig. $1 \mathrm{~b} ; \varepsilon_{\mathrm{p}}$ and $\varepsilon_{\mathrm{w}}$ are the effective permittivity of the upper layer and lower nanowired layer respectively. The internal and external inductances [5] are taken into account in the permittivity of the nanowired layer:

$$
\varepsilon_{w}=p\left(1-j \frac{\sigma_{w} \cdot R_{d c}}{\omega \varepsilon_{0} Z_{t o t}}\right)+(1-p) \varepsilon_{b}\left(1-j \cdot \tan \delta_{b}\right)
$$

with $p$, the porosity of the membrane, $\sigma_{\mathrm{w}}$ the wire conductivity, $\mathrm{R}_{\mathrm{dc}}$ the nanowire resistivity when no external field is applied, $\tan \delta_{\mathrm{b}}$ the dielectric loss tangent factor of the bulk polymer and $\varepsilon_{\mathrm{b}}$ the bulk permittivity. $Z_{\text {tot }}=R d c+j \omega h_{\text {wire }} \mu_{\text {int,eff }} / 8 \pi+j \omega L_{\text {ext }}$ is the total impedance of the wires, depending on the effective internal permeability $\mu_{\text {int,eff }}$ and the external inductance of the wires [4]. With this modeling, the permittivity of the two-layer topology, corresponding to incomplete filling of the nanowires $\left(h_{w}=h_{p}-h_{a}<h_{p}\right)$, is given by expression (2), while expression (3) gives the permittivity for the one-layer substrate, for which $\varepsilon_{\text {eff }}=\varepsilon_{w}$, since $h_{a}=h_{p}-h_{w}=0$. Fig. 3 a shows the effective permittivity for the two-layer substrate and Fig. $3 \mathrm{~b}$ the result for the one-layer substrate. The large difference of magnitude between the two models can be explained by the fact that the one-layer substrate is governed by equation (3), where the first term is proportional to the wire conductivity. It will largely dominate and yields to a highly negative value of the real part, while in the two-layer approach given by equation (2), the term $h_{p} /\left(h_{a} / \varepsilon_{p}\right)$ dominates, which is several orders of magnitude lower. The permittivity for the one-layer substrate is negative for "low frequencies", as predicted by Pendry for artificial materials containing arrays of wires [6]. The magnetodielectric behavior is visible in Fig. 3b. It occurs when porosity and nanowire height are high enough. It is characterized by the fact that the effective permittivity becomes positive in a small window in the microwave range. In the result of the two-layer substrate it is not visible; it occurs as well but is masked by the big influence of the air capacitance between the top of the nanowire and the bottom of the microstrip line. The reason is again the high value of the conductivity in equation (3), making term $\left(\mathrm{h}_{\mathrm{a}} / \varepsilon_{\mathrm{p}}\right)$ dominant in denominator of expression (2). 


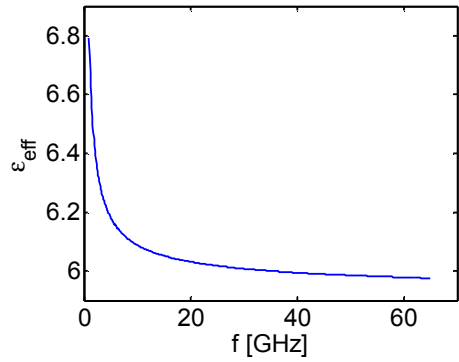

(a)

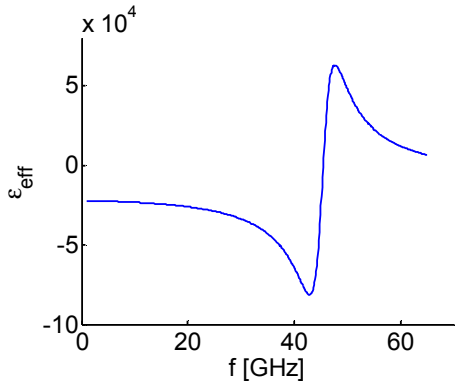

(b)

Fig. 3: (a) Simulation of effective permittivity for two-layer substrate, where $p=20 \%$ and the nanowire height is $60 \%$ of the substrate height, and (b) for one-layer substrate, $\mathrm{p}=20 \%$, the one layer has the same height as the nanowired layer in the two-layer substrate.

Another characteristic of ferromagnetic nanowired substrates is their tunability by applying external magnetic fields. Fig. 4a shows this behavior for the effective permeability of a single wire and Fig. $4 \mathrm{~b}$ the effective permittivity for the one-layer substrate. The wire permeability (Fig. 4a) plays here an important role, since the magnetodielectric effect is correlated to its variation. It is visible that there occurs a zone where both permeability and permittivity are negative.

It should be underlined that the effective permeability of the two-layer substrate shown in Fig 2 does not show any negative value, because of the low porosity. But if the porosity and the wires are high enough [2], we can obtain a negative effective permeability, needed to create double negative metamaterials.

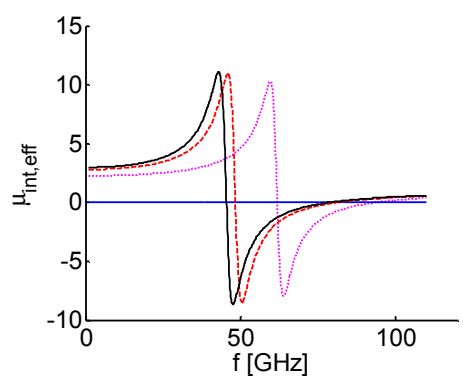

(a)

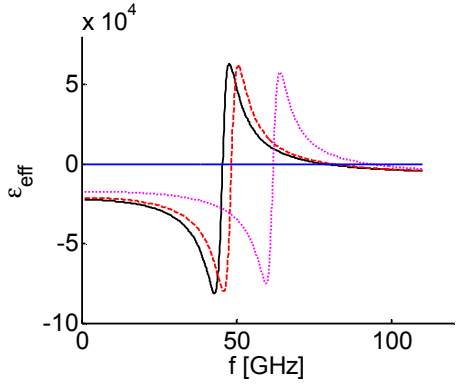

(b)

Fig. 4: Simulation of (a) internal effective permeability $\mu_{\text {int,eff }}$ of wires and (b) effective permittivity of onelayer substrate with applied external magnetic field, 0Oe (solid), $6 \mathrm{kOe}$ (dashed) and $10 \mathrm{kOe}$ (dotted), $p=20 \%$, same height as in Fig. $3 b$.

\section{Conclusion}

In this paper we have studied the permittivity of a one- and two-layer substrate filled with ferromagnetic nanowires. The magnetodielectric effect occurs in these nanowired composite substrates induced by magnetic properties of the wires, but is only visible for the one-layer substrate. It was observed that under specific conditions, especially porosity and filling height of wires, a tunable magnetodielectric effect can be obtained. Furthermore a proper choice of the porosity should enable to create double negative substrates ( $\varepsilon$ and $\mu$ negative) enabling left-handed propagation for miniaturized Magnetic/Dielectric Electromagnetic Bandgap structures based on periodic substrates [3], [7]. 


\section{References}

[1] I. Huynen, G. Goglio, D. Vanhoenacker, and A. Vander Vorst, IEEE Microw. Guided Wave Lett., vol. 9, no. 10, pp. 401-403, Oct. 1999.

[2] J. Spiegel, J. de la Torre, M. Darques, L. Piraux, and I. Huynen, IEEE Microwave and Wireless Components Letters, vol.17, no. 7, July 2007.

[3] J. Spiegel, L. Piraux and I. Huynen, Proceedings of the $37^{\text {th }}$ European Microwave Conference, October 8-12 2007.

[4] A. Berk, IEEE Transactions on Antennas and Propagation, vol. AP-4, pp. 104-111, April 1956.

[5] V. Boucher and D. Ménard, Journal of Applied Physics, vol. 103, 07E720, 2008.

[6] J. B. Pendry et al., Phys Rev. Lett., vol. 76, no. 25, pp. 4773-4776, 1996.

[7] A. Saib, D. Vanhoenacker, I. Huynen, A. Encinas, L. Piraux, E. Ferain, R. Legras, Applied Physics Letters, vol. 83, no. 12, pp. 2378-23, September 2003. 